Please do not remove this page

RMIT

UNIVERSITY

On the numerical study of bubbly wakes generated by ventilated cavity using population balance approach

Xiang, M; Cheung, Chi Pok; Tu, Jiyuan; Zhang, W. H.

https://researchrepository.rmit.edu.au/esploro/outputs/9921857955401341/filesAndLinks?institution=61RMIT_INST\&index=null

Xiang, M., Cheung, C. P., Tu, J., \& Zhang, W. H. (2010). On the numerical study of bubbly wakes generated by ventilated cavity using population balance approach. Journal of Computational Multiphase Flows, 2(2), 101-117. https://doi.org/10.3166/ria.24.291-345

Document Version: Published Version

Published Version: https://doi.org/10.3166/ria.24.291-345

Repository homepage: https://researchrepository.rmit.edu.au

(c) 2010 Multi-Science Publishing Co. Ltd.

Downloaded On 2023/04/26 13:59:56 +1000 
Thank you for downloading this document from the RMIT Research Repository.

The RMIT Research Repository is an open access database showcasing the research outputs of RMIT University researchers.

RMIT Research Repository: http://researchbank.rmit.edu.aul

\section{Citation:}

Xiang, M, Cheung, C, Tu, J and Zhang, W 2010, 'On the numerical study of bubbly wakes generated by ventilated cavity using population balance approach', Journal of Computational Multiphase Flows, vol. 2, no. 2, pp. 101-117.

See this record in the RMIT Research Repository at:

https://researchbank.rmit.edu.au/view/rmit:12712

Version: Published Version

\section{Copyright Statement:}

(C) 2010 Multi-Science Publishing Co. Ltd. Creative Commons Attribution 4.0 International License.

\section{Link to Published Version:}

http://www.ingentaconnect.com/content/doaj/1757482x/2010/00000002/00000002/a rt00003 


\title{
On the Numerical Study of Bubbly Wakes Generated by Ventilated Cavity using Population Balance Approach
}

\author{
M. Xiang ${ }^{1,2}$, S. C. P. Cheung ${ }^{2}$, J. Y. Tu ${ }^{2}$ and W.H. Zhang ${ }^{1}$ \\ ${ }^{1}$ Institute of Aerospace and Material Engineering, National University of Defense \\ Technology, Changsha 410073, P.R. China \\ e-mail: xiangmin333@hotmail.com \\ ${ }^{2}$ School of Aerospace, Mechanical and Manufacturing Engineering, RMIT University, \\ Victoria 3083, Australia \\ e-mail: chipok.cheung@rmit.edu.au; jiyuan.tu@rmit.edu.au
}

Received: 1 June 2010, Accepted: 2 July 2010

\begin{abstract}
In this study, an Eulerian-Eulerian two-fluid model integrated with the population balance approach based on Multiple-Size-Group (MUSIG) model was proposed to simulate on the gas leakage bubbly wake of a ventilated cavitation problem. Three selected flow conditions with Froude number ranging from 20 to 29 have been selected for investigation. Predicted void fraction and bubble velocity profiles were validated against the experimental measurements in the high-speed water tunnel of Schauer (2003) and Wosnik (2005). Sensitivity studies on the mesh resolution and three different turbulence closures were first carried out. In comparison with experimental data, the shear stress transport (SST) turbulence model was found to be the best candidate in modelling the re-circulation motions within the cavity wake region. To consider the neighbouring effect of closely packed bubbles, an empirical equation was proposed to correlate the turbulent dispersion coefficient to the local gas void fraction. Based on the proposed empirical equation, the turbulent dispersion coefficient reduces to 0.1 when local gas void fraction is higher than $60 \%$. In general, numerical predictions were in satisfactory agreement with the experimental data. Some discrepancies have nonetheless been found between the numerical and experimental results. The lack of exact gas leakage mechanism remains an outstanding challenge in determining the actual gas leakage rate and initial bubble size from the continuous cavity. Further effort should be also focused on combing free-surface tracking model with the present population balance approach to investigate the complex vortex structure and interaction between ventilated cavity and discrete leakage bubbles.
\end{abstract}

Keywords: Ventilated cavity, bubbly flow, numerical simulation, population balance, MUSIG

\section{INTRODUCTION}

Natural cavitation occurs in liquid when liquid local pressure is lower or near its vapor pressure. Cavitation number, a special form of Euler number, is a crucial parameter for cavitation analysis. Decrease of cavitation number will produce more vapor bubbles in cavitating zone. With a sufficiently low cavitation number, large amount vapor bubbles will be generated forming a continuous cavity covering the entire underwater object. This particular phenomenon is called supercavitation. In recent years, attracted by its great potential in drag reduction and vehicle manoeuvring applications, various research works have been carried to investigate the complex physical behaviour of supercavitation (David, 2001, Hrubes, 2001, Kuklinski et al. 2001, Semenenko, 2001). In general, two different methods can be adopted to achieve supercavitation. One of the approaches is to increase the liquid dynamic pressure by increasing vehicle 
manoeuvring velocity. Nevertheless, it is rather difficult to maintain such high speed due to the friction of liquid. Another approach is to increase the cavity pressure by injecting gas bubble into the liquid. This method is commonly referred as - ventilated cavitation.

One critical problem for ventilated cavitation has been to determine the amount of ventilation gas that is required to sustain a supercavity for a given vehicle or dimension. At steady state, without any phase change due to condensation or evaporation, gas ventilation rate is balanced by the gas leakage rate at the cavity tail. According to Semenenko (2001), if the cavity deformation due to gravity effect is insignificant, gas leakage is primarily governed by toroidal vortex shedding structure and recirculation motions of the re-entrained jets. As depicted in Figure 1, caused by the high shear stress, the vortex flow structure shatters the gas cavity and exposes part of the vehicle body to liquid phase. This particular shape of cavity is commonly referred as Gilbarg-Efros type cavity closure.

It is well known that the Gilbarg-Efros type cavity could induce unsteady hydrodynamic forces, structural erosion and vibration problems to the vehicle body. In order to circumvent this problem, a fundamental understanding of the two-phase flow structure and its associated gas leakage mechanism at various flow conditions is crucial. Unfortunately, it remains an extreme challenge to comprehend the exact mechanism not only in regard to the presence of discrete interfaces with large density ratio; but also the morphology transition from continuous cavity to dispersed gas bubbles.

A number of previous studies have been carried out attempting to investigate such complex physical phenomenon. Young and Kinnas (2003) employed potential flow method to model toroidal vortex shedding structure. Nevertheless, aiming to simplify the computational scheme, interfacial viscous effect and dynamics of the free-surface were ignored in their simulation. The method also heavily relied on the empirical models for cavity closure; where gas leakage processes were not solved explicitly. Recently, a more sophisticated modelling approach based on EulerianEulerian framework was widely adopted (Owis, 2002; Qin and Song, 2003; Kunz and Lindau, 2000, 2005; Goncalves, 2009). Qin and Song (2003) proposed a density mixture model to simulate the flow structure of ventilated cavitation. Similar numerical scheme was also found in Goncalves (2009). In their model, flow field was solved based on one set of continuity and momentum equations. Density of the fluid was evaluated based a continuous function expressed in terms of gas void fraction which is solved by an additional transport equation. However, interfacial effect of the free-surface was also neglected. Kinzel $(2007,2008)$ adopted the Volume-Of-Fluid (VOF) model to explicitly track free-surface of the cavity. Although the free-surface was successfully captured by the VOF, noticeable prediction errors were reported for the predictions in the wake region where continuous cavity was shattered into dispersed gas bubbles.

In summary, aforementioned studies were primarily focused on capturing free-surface interface of the front part of the ventilated cavity. Re-circulated motions and dispersion of discrete gas bubbles in the wake region have received less attention in literatures. One should be noticed that the breakdown of cavity is predominated by toroidal vortex structure and dispersion of bubbly flow in the wake region. To better understand the Gilbarg-Efros type cavity closure, numerical simulations using Eulerian-Eulerian two-fluid model were carried out to model the dispersion of discrete gas bubble and its embedded complex flow structures. Population balance approach based on the MUltiple-SIze-Group (MUSIG) model (Olmos et al., 2001; Yeoh and Tu, 2004, 2005; Frank et al., 2005) was adopted to capture the evolution of the bubble size distribution due to coalescence and breakage. Numerical predictions were then validated against the re-entrained jet experiment by Wosnik et al. (2003 and 2005).

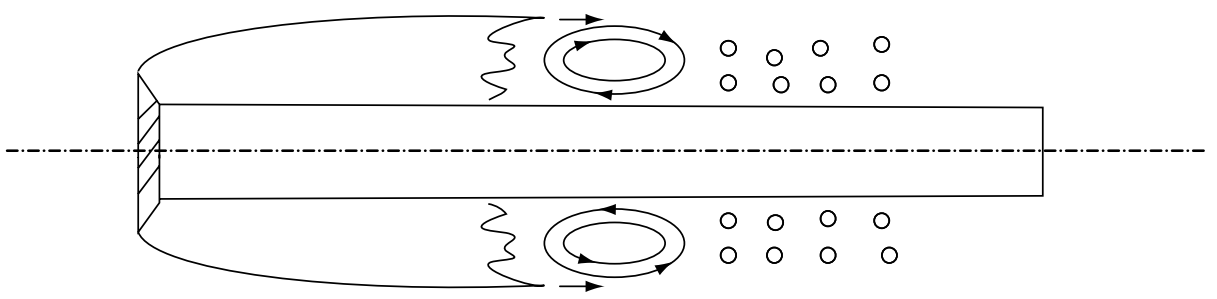

Figure 1. Schematic of toroidal vortex shedding structure and re-entrained jet of a Gilbarg-Efros type cavity closure. 


\section{MATHEMATICAL MODELS}

\subsection{Two-Fluid Model}

The two-fluid model based on Eulerian-Eulerian approach is one of the widely adopted approaches to model two-phase flows with significant gas void fraction. In this paper, the liquid phase is treated as continuum while the gas phase is considered as dispersed phase. Two sets of governing equations are solved for each phase. As interfacial heat and mass transfer is negligible, energy equation is not included in the numerical scheme.

Without interfacial mass transfer, the continuity equation of the two-phases can be written as:

$$
\frac{\partial\left(\rho_{i} \alpha_{i}\right)}{\partial t}+\nabla \cdot\left(\rho_{i} \alpha_{i} \overrightarrow{u_{i}}\right)=0
$$

where $\alpha, \rho$ and $\vec{u}$ is the void fraction, density and velocity of each phase. The subscripts $i=l$ or $g$ distinguish the liquid and gas phase.

The momentum equation for the two-phase can be expressed as follows:

$$
\frac{\partial\left(\rho_{i} \alpha_{i} \overrightarrow{u_{i}}\right)}{\partial t}+\nabla \cdot\left(\rho_{i} \alpha_{i} \overrightarrow{u_{i}} \overrightarrow{u_{i}}\right)=-\alpha_{i} \nabla P+\rho_{i} \alpha_{i} \vec{g}+\nabla \cdot\left[\alpha_{i} \mu_{i}^{e}\left(\nabla \overrightarrow{u_{i}}+\left(\nabla \overrightarrow{u_{i}}\right)^{T}\right)\right]+F_{i}
$$

where $\mu^{e}$ refers to the effective viscosity which is summation of dynamics viscosity and turbulent viscosity, $\vec{g}$ is the gravity acceleration vector. $F_{i}$ represents the total interfacial drag and non-drag forces which govern the distribution of the liquid and gas phase distribution. Details of the interfacial terms are described as follows.

\subsection{Interfacial Momentum Transfer}

The total interfacial forces can be categorized into four main terms: drag, lift, wall lubrication and turbulence dispersion. Based on extensive parametric study, the effect of wall lubrication forces has been found to exert insignificant influence to the gas phase distribution. Wall lubrication forces are therefore neglected. The total interfacial forces are then given by:

$$
F_{i}=F_{1 g}=-F_{1 g}=F_{1 g}^{\text {drag }}+F_{1 g}^{l i j t}+F_{1 g}^{\text {dispersion }}
$$

Here, $F_{l g}$ denotes the momentum transfer terms from the gas phase to the liquid phase and vice versa for $F_{g l}$.

\subsubsection{Drag Force}

The inter-phase momentum transfer between gas and liquid due to the drag force is modelled according to Ishii and Zuber (1979) as:

$$
F_{l g}^{\text {drag }}=-F_{g l}^{\text {drag }}=\frac{1}{8} C_{D} a_{i f} \rho_{l}\left|u_{g}-u_{l}\right|\left(u_{g}-u_{l}\right)
$$

where $C_{D}$ is the drag coefficient which can be evaluated by correlation of several distinct Reynolds number regions for individual bubbles. The model also takes into account dense particle effects, which is suitable for the multiphase flow in this research containing high void fraction area.

\subsubsection{Lift Force}

Due to velocity gradients in radial and azimuthal directions, bubbles rising in a liquid are subjected to a lateral lift force. Appropriate modelling of the lift force is important to obtain the correct spreading of the gas phase. The force can be correlated to the relative velocity and the local liquid vorticity from Drew and Lahey (1979) as:

$$
F_{l g}^{l i f t}=-F_{g l}^{l i f t}=C_{L} \rho_{l}\left(\overrightarrow{u_{g}}-\overrightarrow{u_{l}}\right) \times\left(\nabla \times \overrightarrow{u_{l}}\right)
$$


For the lift coefficient, $C_{L}$, the EÖtvos number dependent correlation proposed by Tomiyama (1998) is adopted. More details can be found in Cheung et al. (2007).

\subsubsection{Turbulent Dispersion Force}

To account for the random (dispersive) influence of the turbulent eddies on the bubbles, the concept of the turbulent dispersion force is introduced. The effect of the turbulent dispersion force, just like that of the lift force, is also important to obtain correct dispersion of the gas. The turbulent dispersion force expression in terms of Farve-averaged variables proposed by Burns et al. (2004) is adopted, viz:

$$
F_{l g}^{d i s p e r s i o n}=-F_{g l}^{d i s p e r s i o n}=C_{T D} C_{D} \frac{v_{t, g}}{\sigma_{t, g}}\left(\frac{\nabla \alpha_{l}}{\alpha_{l}}-\frac{\nabla \alpha_{g}}{\alpha_{g}}\right)
$$

where $C_{T D}, C_{D}, v_{t, g}$ and $\sigma_{t, g}$ represent the turbulent dispersion coefficient, drag force coefficient, turbulent kinematic viscosity for the gas phase and the turbulent Schmidt number of the gas phase, respectively. By default, the turbulent dispersion coefficient $C_{T D}=1$ and the turbulent Schmidt number $\sigma_{t, g}=0.963$ are adopted.

\subsection{Population Balance Model}

The population balance method is adopted to predict the size distribution of the dispersed bubbles. The population balance equation is solved by the Multiple-Size-Group (MUSIG) model where bubble size distribution is discretized with multiple discrete bubble classes. Assuming each bubble class travel at the same mean algebraic velocity, individual number density of bubble class $i$ based on Kumar and Ramkrishna (1996a) can be expressed as:

$$
\frac{\partial n_{i}}{\partial t}+\nabla \cdot\left(\overrightarrow{u_{g}} n_{i}\right)=P_{c}+P_{B}-D_{c}-D_{B}
$$

where $n_{i}$ is the average bubble number density of the $i t h$ group, the source terms $P_{C}, P_{B}, D_{C}$ and $D_{B}$ are the production rates due to coalescence and break-up and the death rate due to coalescence and break-up of bubbles respectively. The break-up rate of bubbles is computed according to the model developed by Luo and Svendsen (1996), which is developed based on the assumption of bubble binary break-up under isotropic turbulence situation. The coalescence rate considering only turbulent collision is taken from Prince and Blanch (1990). The breakup and coalescence coefficients are introduced to modify the original correlations which are developed based on bubbly co-flow situation.

\section{EXPERIMENTAL DETAILS}

In the present study, numerical predictions are validated against the experimental measurements in the high-speed water tunnel at St. Anthony Falls Laboratory of the University of Minnesota (Schauer, 2003, Wosnik, 2005). In their experiment, measurements were carried out in the water tunnel test section of $0.19 \mathrm{~m}(\mathrm{~W}) \times 0.19 \mathrm{~m}(\mathrm{H}) \times 1 \mathrm{~m}(\mathrm{~L})$. To study the re-entrained jet leakage mechanism of ventilated cavitation, a test body with $10 \mathrm{~mm}$ cavitator installed at the front was placed at the middle of the test section (see also in Figure 2). Velocity distribution of gas bubbles were measured using Particle Image Velocimetry (PIV) technique. Photographic luminous conversion based the normalized grey-scale value was adopted to extract the time-averaged gas bubble void fraction profiles. Three different flow conditions, expressed in terms of Froude number, dimensionless ventilation rate and cavitation number, are selected for validations. Definitions of Froude number $F_{r}$ and the ventilation rate $\overline{Q_{g}}$ are given:

$$
F_{r}=\frac{u_{\infty}}{\sqrt{g D_{n}}}, \overline{Q_{g}}=\frac{\dot{Q}}{u_{\infty} D_{n}^{2}}
$$

where $u_{\infty}$ refers to the mainstream velocity, $D_{n}$ is the cavitator diameter and $\dot{Q}$ is the volumetric gas ventilation rate. Detailed flow conditions are summarized in Table 1. 


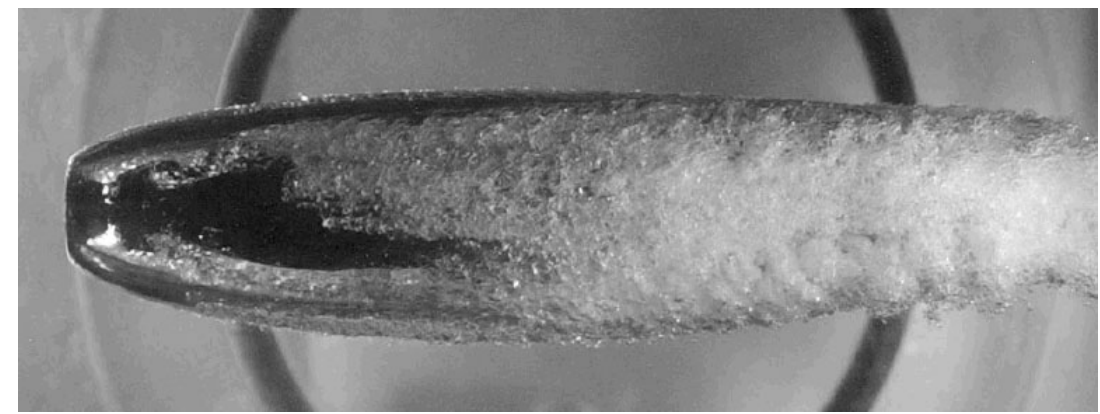

Figure 2. Snapshot of ventilated cavitation and gas leakage behaviour by Wosnik et al. (2003).

Table 1. Flow parameters of the three selected flow conditions for validations

\begin{tabular}{lccc}
\hline & Froude Number, $\boldsymbol{F}_{\boldsymbol{r}}$ & Ventilation rate, $\overline{\boldsymbol{Q}_{\boldsymbol{g}}}$ & Cavitation Number, $\boldsymbol{\sigma}_{\boldsymbol{c}}$ \\
\hline Case $\mathbf{1}$ & 20 & 0 & - \\
Case 2 & 20 & 0.18 & 0.15 \\
Case 3 & 29 & 0.16 & 0.15
\end{tabular}

\section{NUMERICAL DETAILS}

\subsection{Mesh}

Since the experimental water tunnel and test body are symmetric, numerical simulations are only performed on a half portion of the water tunnel. For the non-cavitating flow condition (Case 1), as shown in Figure 5, non-uniform mesh are generated throughout the computational domain covering the whole test body with the cavitator. For the cavitating cases (Case 2 and 3), the front part of the test body is covered by the cavity where gas-liquid interface is assumed to be steady and evaluated according to empirical correlations of Logvinovich (1964) and Savchenko (1998). The cavity is assumed to be ruptured at the highest interfacial shear stress point where the cavity has its maximum diameter. The final outline of test body and the interface of the cavity are depicted in Figure 3. Similar body-fitted grid structures consisting of approximately 160,000 grids are adopted for both non-cavitating and cavitating flows (as shown in Figure 4).

\subsection{Boundary Condition}

For the non-cavitating case, velocity inlet boundary and pressure outlet boundary are applied in accordance with the experimental conditions. The surface of the test body is considered as no-slip boundary. For cavitating cases, as interfacial mass transfer is negligible, gas leakage rate of the cavity is assumed to be equal to the gas ventilation rate as measured in the experiment. Following

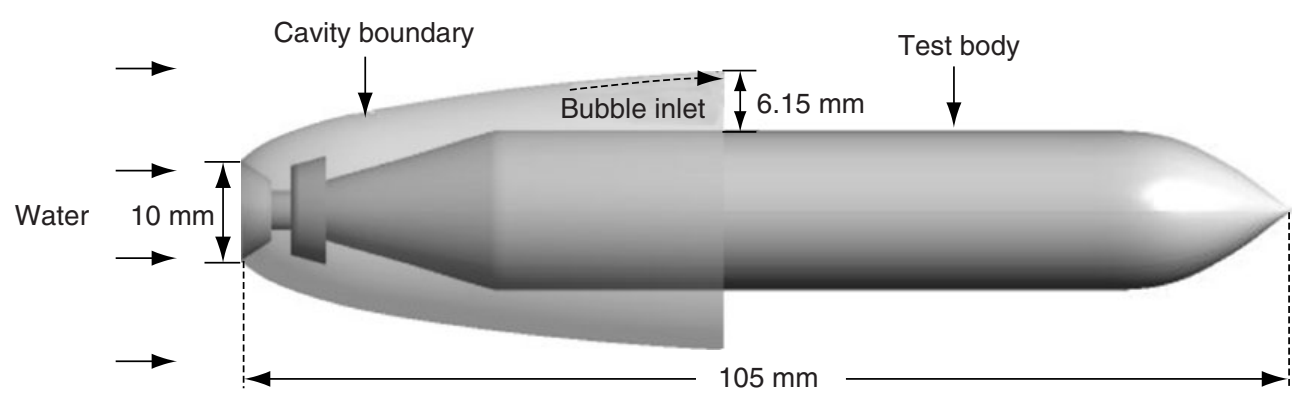

Figure 3. Schematic of the test body with cavity interface evaluated based on Logvinovich (1964) and Savchenko (1998). 


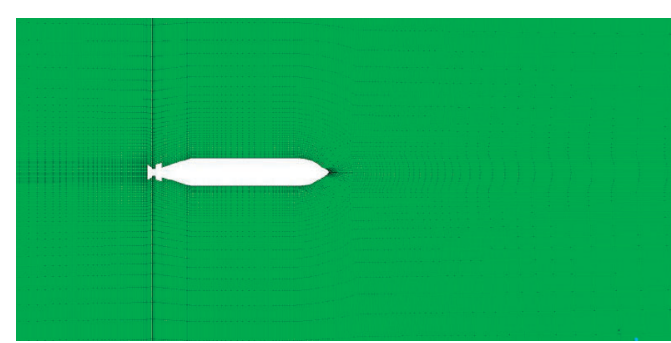

(a) Non-cavitating flow
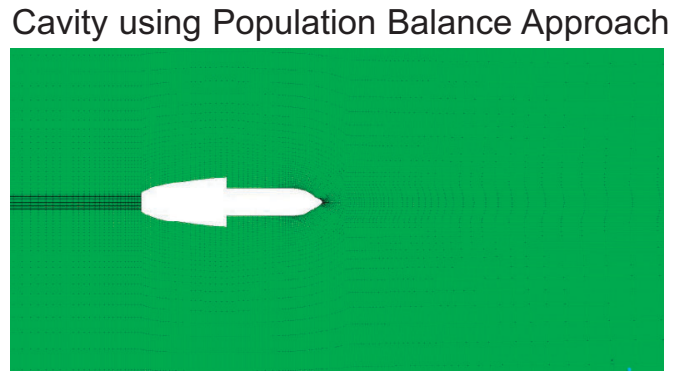

(b) Cavitating flow

Figure 4. Mesh distribution of body-fitted grid structure for the (a) non-cavitating flow and (b) cavitating flow.
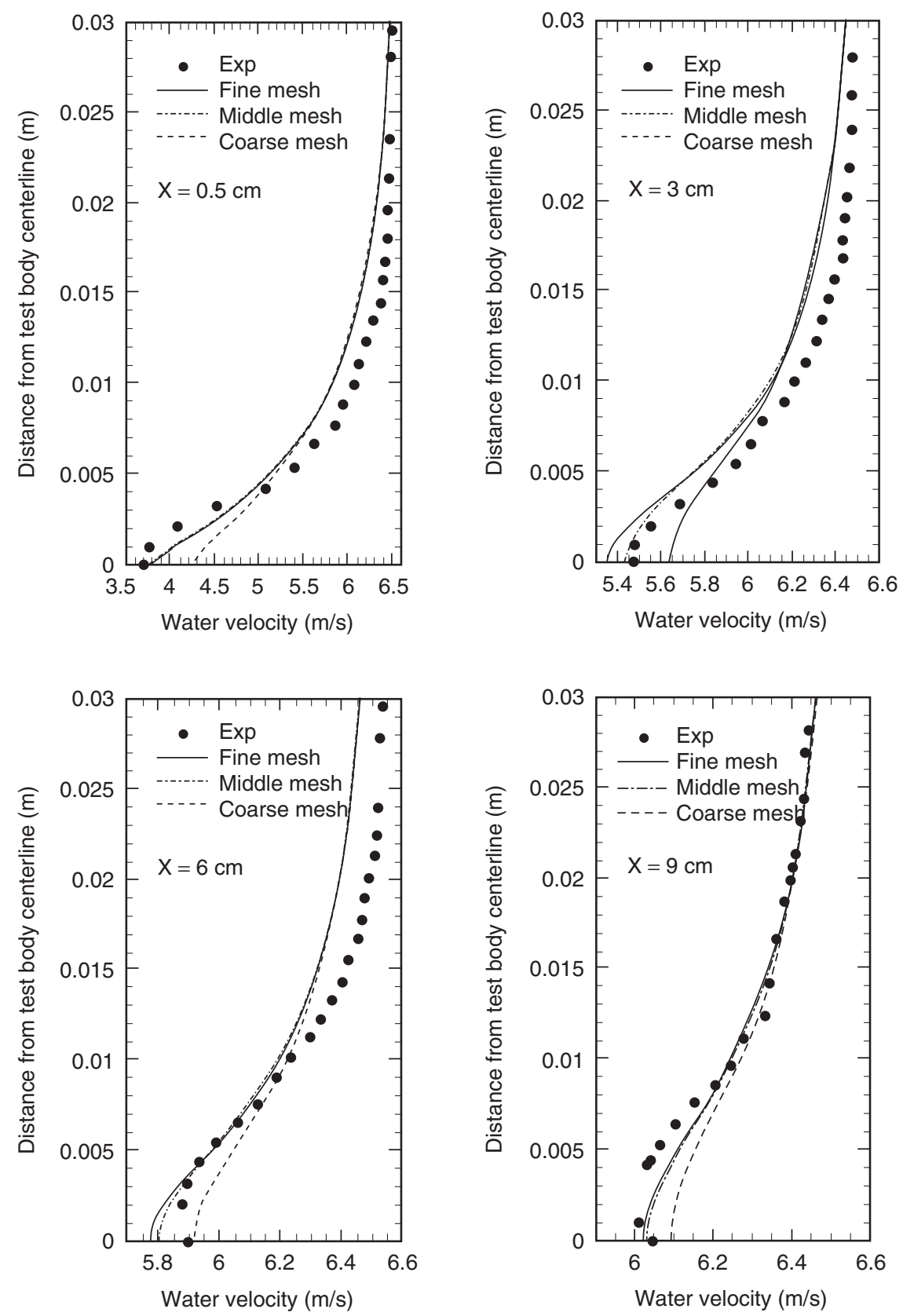

Figure 5. Predicted radial velocity distribution in the wake of the test body using different mesh. 
the study by Evans (2004), the gas leakage rate is specified through a velocity inlet boundary located at the circumference of the cavity base (see also in Figure 3). To determine the area of the inlet boundary, an assumption is made for the gas film thickness. Gas bubbles with uniform diameter are specified at the inlet. Detail parametric studies on the gas film thickness and bubble initial diameter are summarized in section 5.2.1. The cavity interface is set up as free-slip boundary while the test body is modelled as no-slip for both liquid and gas phase.

\subsection{Numerical Method}

The generic CFD code ANSYS CFX 11 (ANSYS, 2005) is employed as a platform for two-fluid flow computation with the built-in MUSIG model adopted. For the MUSIG model, bubbles ranging from 0 to $10 \mathrm{~mm}$ diameter are equally divided into 10 size groups. The governing equations are solved by a finite-volume approach. The convection terms are discretized with second-order upwind scheme while a central difference discretization scheme is applied in the diffusion term. For all flow conditions, reliable convergence is achieved within 2500 iterations when the RMS (root mean square) pressure residual dropped below $1.0 \times 10^{-7}$. A fixed physical time scale of $0.001 \mathrm{~s}$ is adopted for all steady state simulations.

\section{RESULTS}

\subsection{Sensitivity Study on Mesh Resolution and Turbulence Modelling}

In order to carry out grid independence analysis, three grid levels covering the range of 80,000 , 160,000 and 280,000 elements have been created corresponding to coarse, medium and fine mesh. Figure 5 shows the predicted radial velocity distribution obtained using different mesh levels at various downstream distances in comparison to the experimental data. As depicted, velocity magnitude within the wake gradually reaches its freestream value as the downstream distance increases. In general, the asymptotic behavior of the axisymmetric wake is accurately captured by all levels of grid. Almost identical predictions are observed between middle and fine mesh at all four downstream positions. Therefore, it can be concluded that the middle mesh resolution is sufficient for obtaining grid independent solutions. The $\mathrm{y}^{+}$values at the centre of the first cell of the middle mesh vary between 12 and 27.

Sensitivity study on the turbulence closure was also carried out. Radial velocity distributions predicted by three different turbulence models (i.e. standard $k \sim \varepsilon$ model, RNG $k \sim \varepsilon$ model and the $k \sim \omega$ based Shear Stress Transport (SST) model (Menter, 1994)) were compared with experimental data. As shown in Figure 6, the standard $k \sim \mathcal{E}$ model over-predicted the velocity magnitude; while predictions of the SST model were in good agreement with measurements. As a result, all numerical predictions presented hereafter were obtained from the middle mesh and SST turbulence model.

\subsection{Multiphase Model Analysis}

\subsubsection{Parametric Studies on the Gas Film Thickness and Initial Bubble Diameter}

For cavitating cases, as the interface of the cavity is pre-determined by empirical correlations, gas leakage rate is modelled by specifying an inlet boundary at the tips of the cavity base. One parameter determining the gas inlet area is the gas film thickness $\delta_{g}$. As there is no measurement made on the gas film thickness, a parametric study has been carried out to investigate its influence to the downstream gas void fraction distribution.

The parametric study was carried out with respect to the cavitating Case 2. Figure 7 shows the predicted void fraction distribution along the surface of the test body at the downstream of the inlet based on two $\delta_{g}$ values (i.e. $1 \mathrm{~mm}$ and $5 \mathrm{~mm}$ ). As depicted, the two selected gas film thickness have insignificant influence on the void fraction distribution. In both film thicknesses, gas void fraction became zero at the same axial distance $\mathrm{x}=0.082 \mathrm{~m}$. This demonstrated that the predicted liquid reattachment points were practically identical in regardless the gas film thickness. Void fraction values beyond the liquid re-attachment point were almost identical in both cases.

The initial bubble diameter specified at the inlet boundary is another important parameter to model the gas leakage behaviour. Figure 5 shows the profile of the sauter mean bubble diameter along the radial direction at the first grid cell downstream of the cavity base. As depicted, three initial bubble diameters, corresponding to 1,3 and $8 \mathrm{~mm}$ respectively, were specified at the inlet boundary. Nevertheless, for other downstream locations, differences of the bubble diameter were significantly diminished. This is due to the fact that the downstream bubble sizes were 


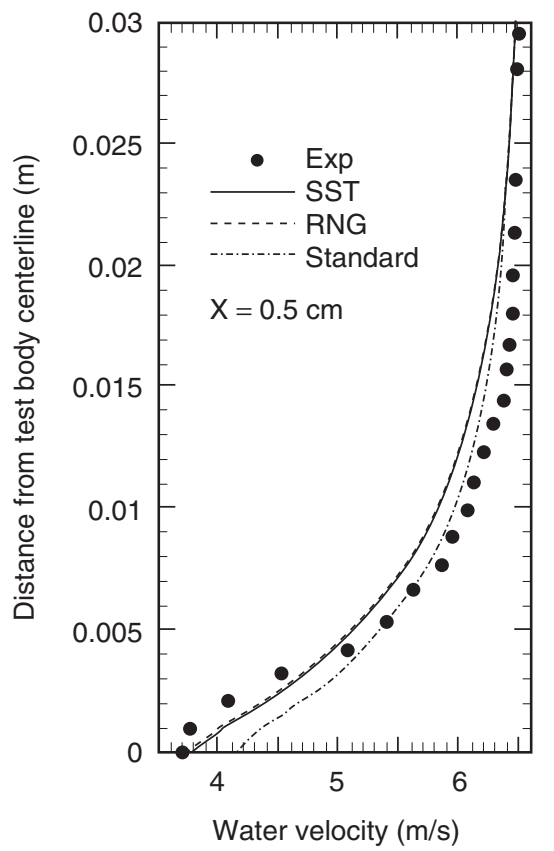

Cavity using Population Balance Approach
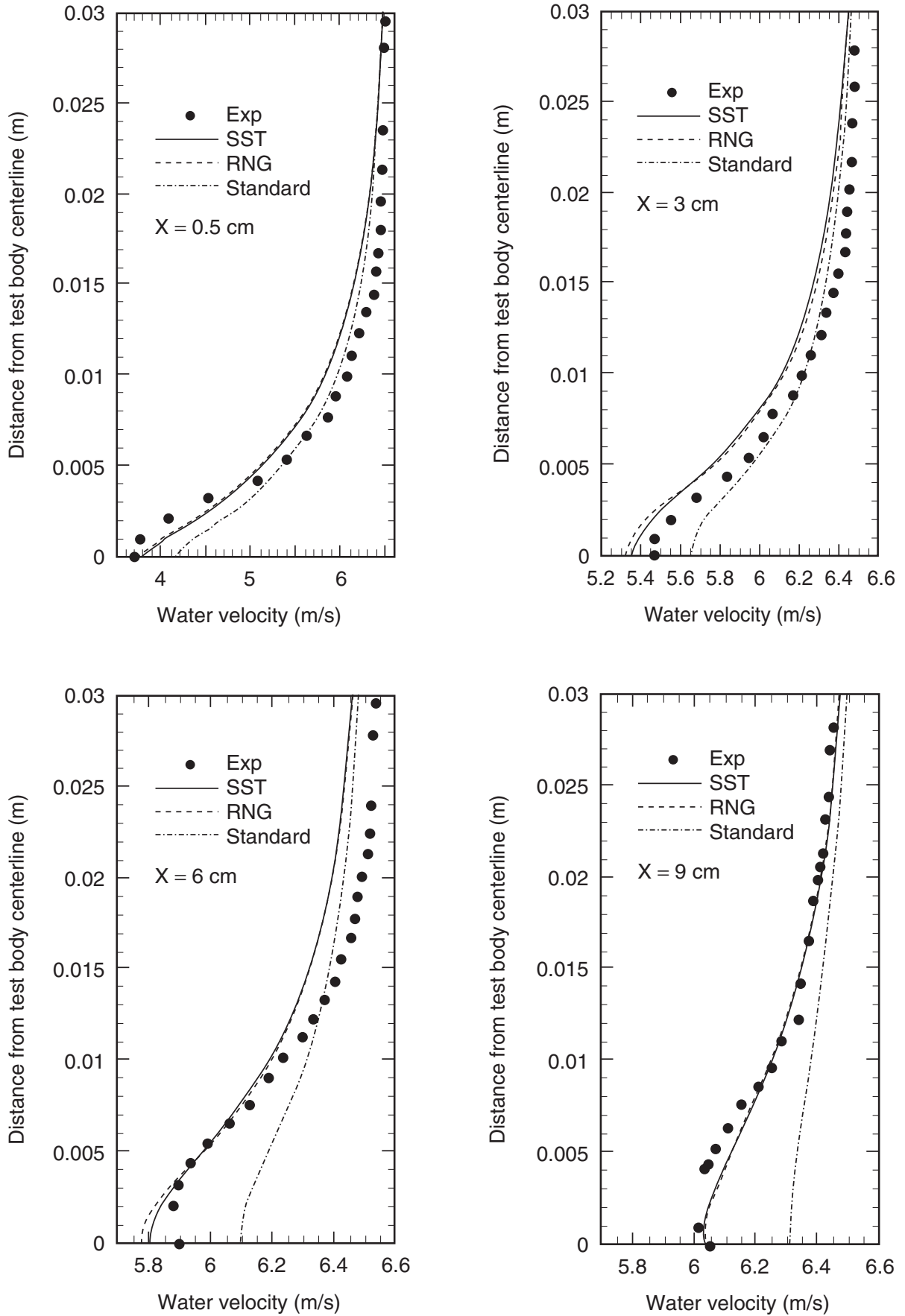

Figure 6. Predicted radial velocity distribution in the wake of the test body using different turbulence model.

predominately governed by the coalescence and breakage mechanism; of which are mainly related to the local turbulent dissipation rate. The above parametric studies demonstrated that the assumption of the gas film thickness and the initial bubble diameter have insignificant influence on the main torodial re-entrained jet structure. Discussions and findings presented hereafter obtained with the gas film thickness of $1 \mathrm{~mm}$ and the initial bubble diameter of $3 \mathrm{~mm}$.

\subsubsection{Turbulent Dispersion Force}

Turbulent dispersion force plays a significant role in the present study, due to its dominant effect on the dispersion of discrete gas bubbles. Preliminary simulation results for the Case 2 using two 


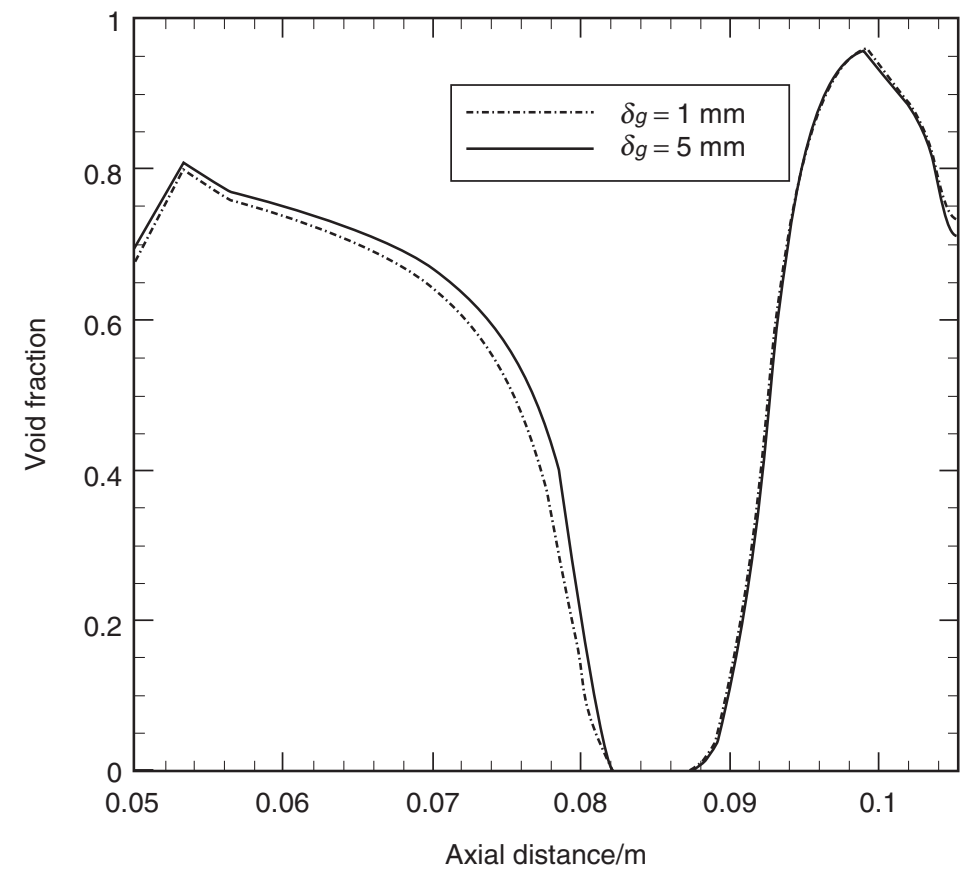

Figure 7. Void fraction distribution along the wall of the test body with different gas film thickness.

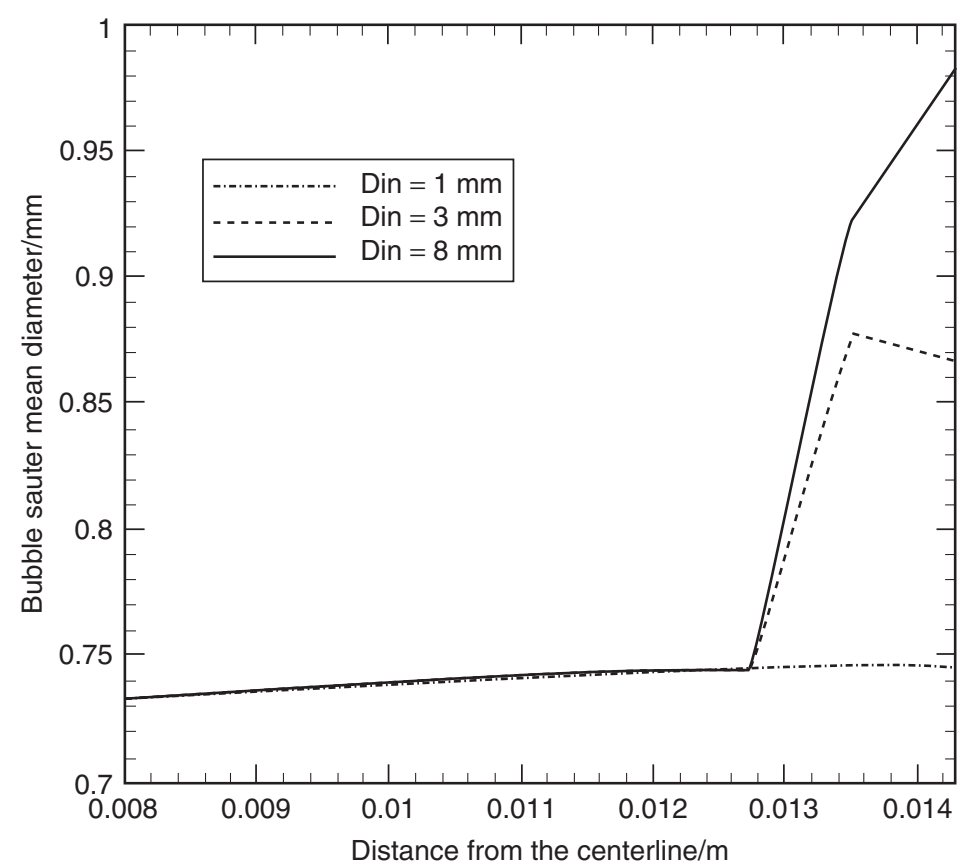

Figure 8. Bubble diameter variation along the gas inlet with different initial bubble size.

different turbulent dispersion coefficient, $C_{T D}$, are presented in Figure 9. As depicted, the void fraction distribution is extremely sensitive to the value of $C_{T D}$. At high $C_{T D}=1.0$, gas void fraction within the wake region behind the cavity were found to be under-predicted. This is due to the fact that the turbulent dispersion rate of discrete bubbles was over-estimated; which in return causing an unrealistic amount of bubble diffused into the mainstream of liquid. However, void fraction distribution along the centre-line behind the test body was found in satisfactory agreement with the experiment data. 


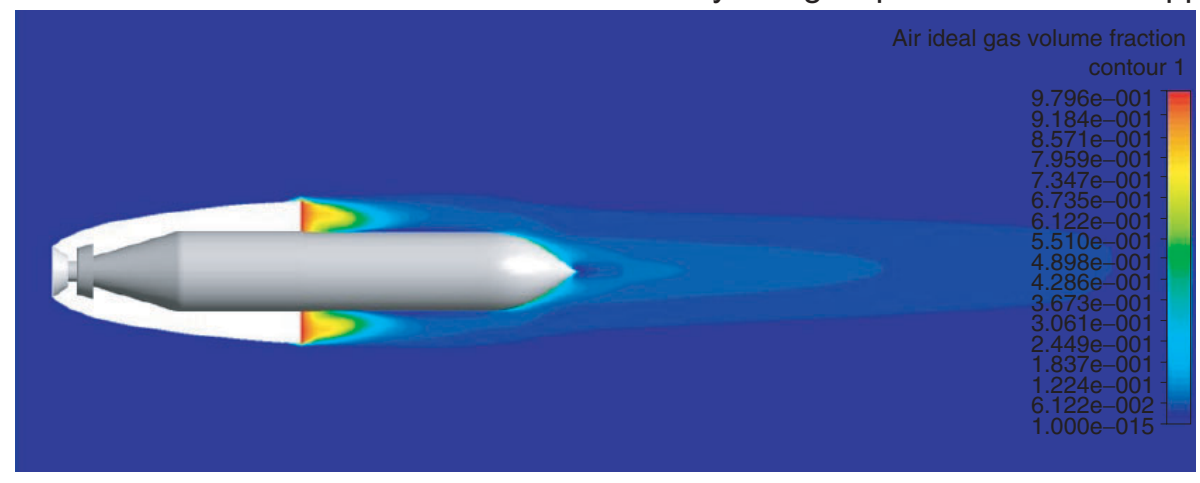

$$
C_{T D}=1
$$

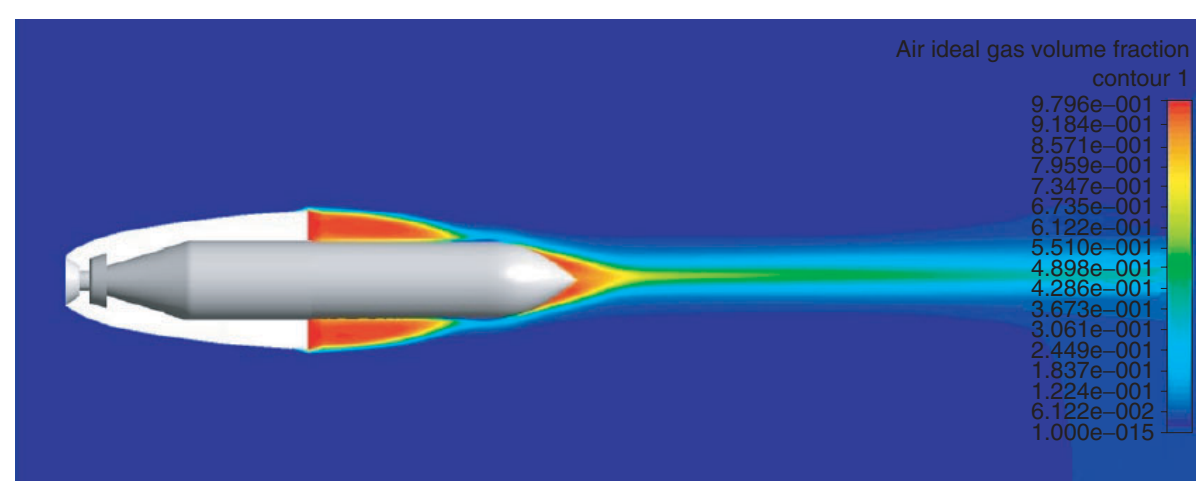

$$
C_{T D}=0.1
$$

Figure 9. Preliminary void fraction contours using different turbulent dispersion coefficients (Case 2).

For low $C_{T D}=0.1$, the void fraction within the wake region was successfully captured; while the void fractions at the downstream of the test body were significantly over-predicted. The above numerical errors were mainly caused by the uniform assumption of the $C_{T D}$. At high void fraction situation, where bubbles are closely packed, turbulent dispersion of bubbles is hindered by the existence of neighbouring bubbles. Similar observations were also found for the drag force of bubble by the experimental study of Simonnet et al. (2007). They concluded that the neighbouring effect of close packed bubbles becomes significant if the void fraction exceeds 15\%. An empirical equation was also proposed to correlate the drag coefficient with the local gas void fraction. Following the same agreement by Simonnet et al. (2007), a new variation law of $C_{T D}$ is developed to take into account of the effect of void fraction as follows:

$$
C_{T D}=C_{\max }\left(1-\alpha_{g}\right)\left[\left(1-\alpha_{g}\right)^{2}+\left(m \frac{\alpha_{g}}{1-\alpha_{g}}\right)^{2}\right]^{-1}+C_{\text {min }}
$$

This formula contains 3 main empirical constants (i.e. $C_{\min }, C_{\max }$ and $m$ ). $C_{\min }$ and $C_{\max }$ determine the minimum and maximum turbulent dispersion coefficient respectively; while $m$ is the criterion for dividing low and high void fraction region. In our research, $C_{\min }=0.1, C_{\max }=0.9$ and $m=4.8$ are chosen according to the experimental data. The variation of $C_{T D}$ against void fraction as evaluated 


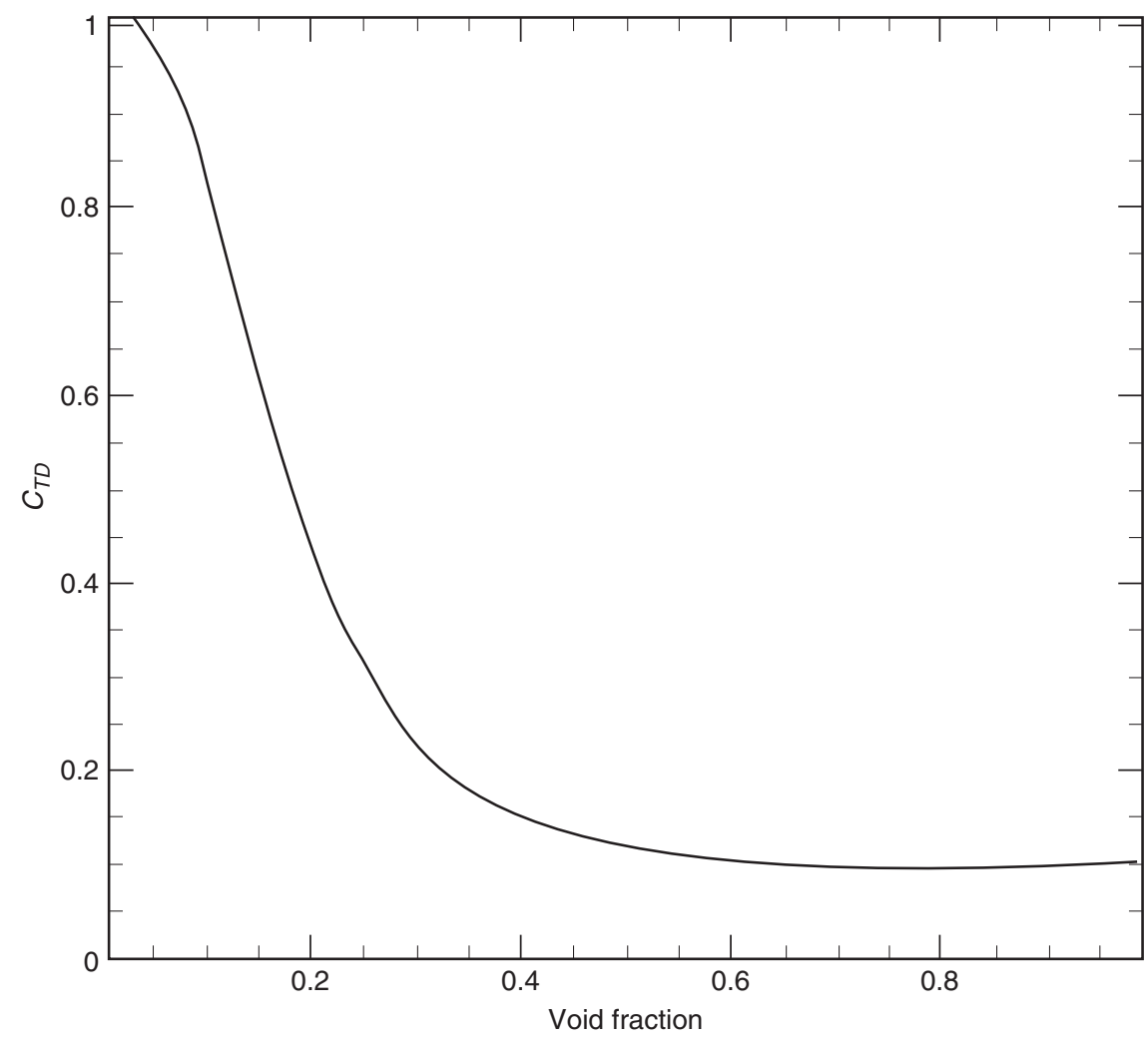

Figure 10. Variation of turbulence dispersion coefficient against void fraction.

according to Equation 9 is shown in Figure 10. As depicted, $C_{T D}$ decreases dramatically when void fraction is higher then 0.05 . When void fraction reaches around the distinguishing point around 0.3 , $C_{T D}$ decreases gently until reaching the minimum value of 0.1 . For high void fraction (i.e. $\alpha_{g} \geq 0.5$ ), $C_{T D}$ is almost constant at its minimum value $C_{T D}=0.1$.

\subsection{Multiphase Flow Characteristics}

\subsubsection{Flow Field Parameters}

The predicted gas void fraction and streamline, pressure and liquid velocity contour at the centreplane of the tunnel water are shown from Figure 11 to Figure 13 respectively. As depicted by the streamline in Figure 11, a strong toroidal vortex structures were formed right behind the cavity base. This re-circulation motion held most of the leakage bubble forming a high void fraction bulk within the wake region. The mainstream of liquid flow was found re-attached to the test body. As additional drag and pressure forces could be imposed on the test body (see also in Figure 12), possible causing structural vibration, such re-attachment of liquid flow is not favourable.

Moreover, due the gravity effect, the volume of the lower wake region was found slightly larger than upper wake region. This also contributed to the extra pressure force acting on the lower part of the test body. Figure 14 illustrates the predicted radial void fraction distribution at different axial distances away from the test body in compared to the experimental data. Caused by the bubble dispersion, the maximum void fraction decreased along with axial direction. As depicted, the predicted radial void fraction profiles were in good agreement with the experimental data; denoting that the bubble dispersion has successfully captured by the model. Comparison between the predicted and measured bubble velocity is shown in Figure 15. Mainly driven by the interfacial drag force, bubble velocities were found gradually increase approaching the liquid freestream velocity along axial direction. Although bubbly velocities were slightly under-predicted by the model, the qualitative trends of the velocity profile were successfully captured. 


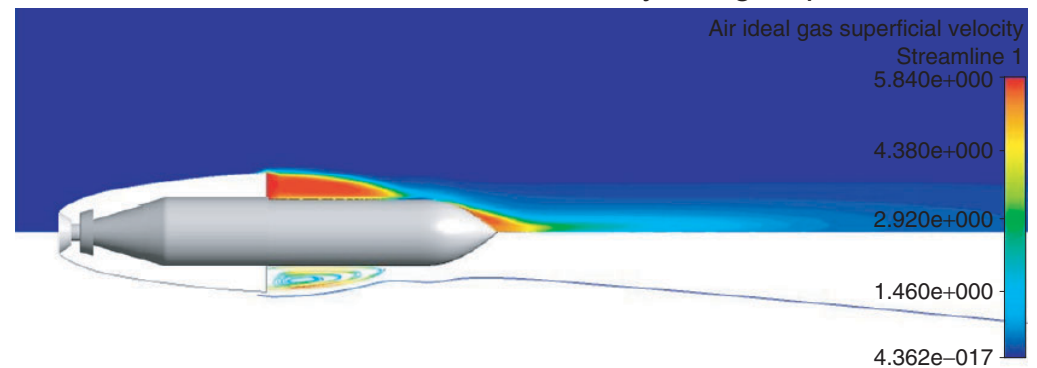

$\left[\mathrm{m} \mathrm{s}^{\wedge}-1\right]$

Figure 11. Void fraction and streamline (Case 2).

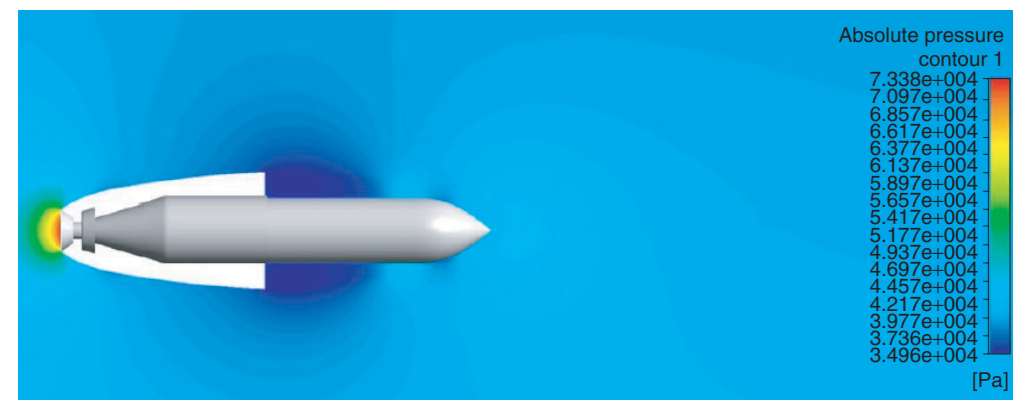

Figure 12. Pressure contours (Case 2).

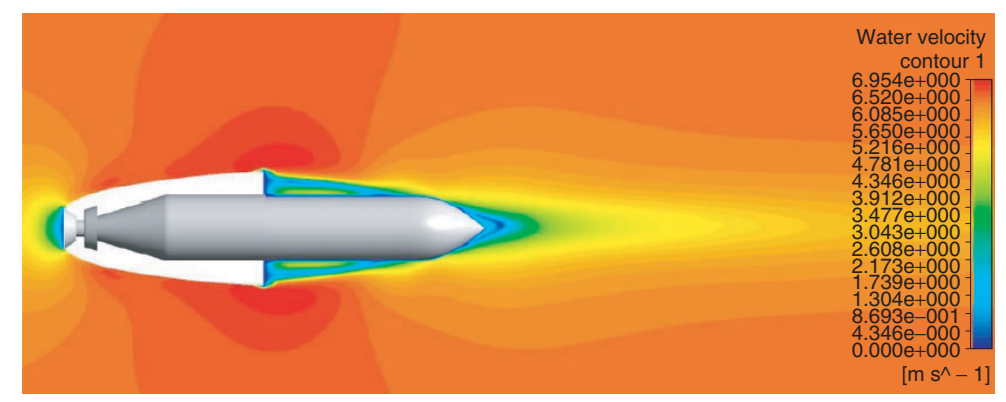

Figure 13. Water velocity contours (Case 2).

\subsubsection{Gravity Effect}

Figure 16 depicts the predicted gas void fraction contour for the cavitating Case 3 . Figure 17 shows a qualitative comparison between predicted and measured void fraction profiles at different axial locations behind the test body. Driven by the gravity effect, the peak of void fraction profiles gradually shifted upper away from the centreline of the test body. It can be observed from the figure that magnitude and trends of void fraction profile were successfully captured by the model. Nevertheless, the locations of void fraction peak were considerably under-predicted. This could be caused by the uniform gas leakage rate imposed at the inlet boundary condition. 


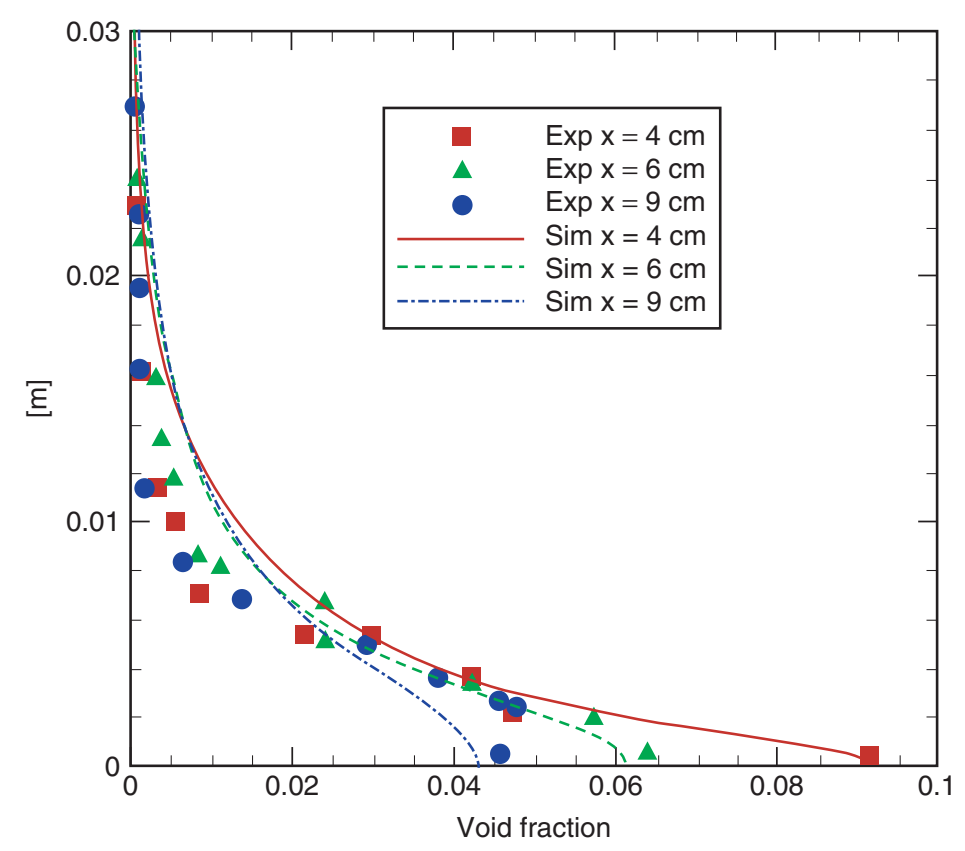

Figure 14. Void fraction in the bubbly wake (Case 2).

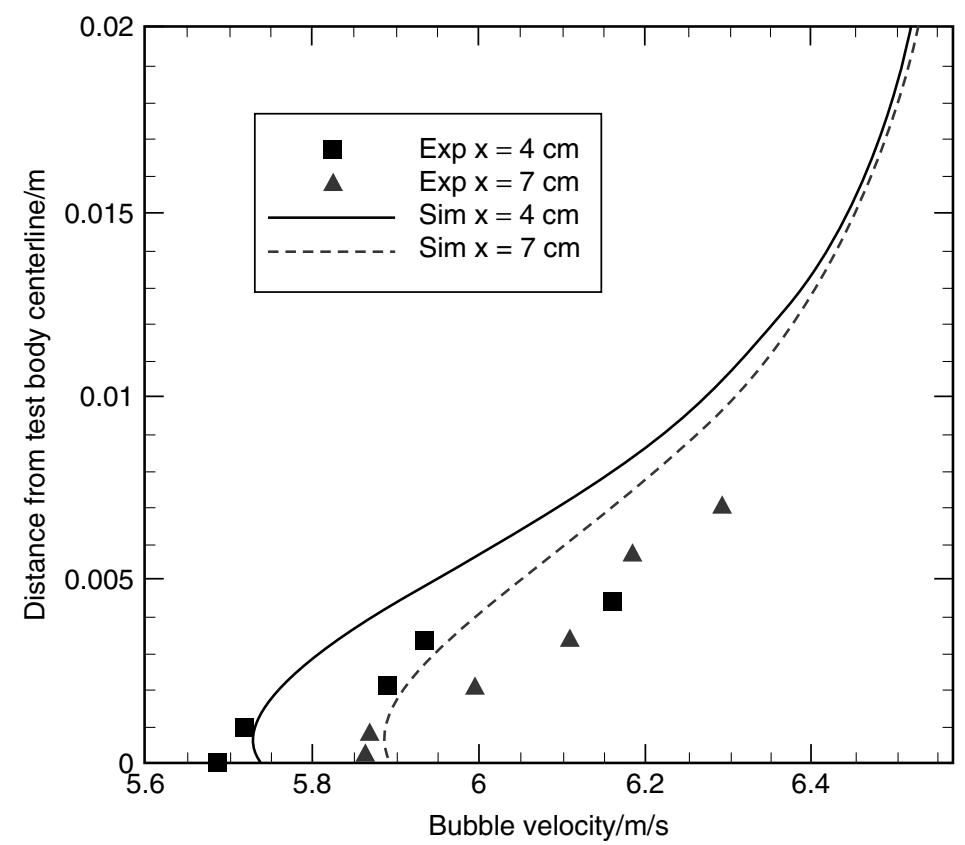

Figure 15. Bubble velocity in the wake (Case 2).

As discussed before, gas leakage mechanism is modelled through the gas injection at the inlet boundary. Assuming the gas leakage rate is identical to the gas ventilation rate, gas injection is uniformly distribution at the inlet boundary in regardless of the gravity effect. However, as confirmed by the experimental observation (Wosnik, 2005), noticeable less amount of gas leakage 


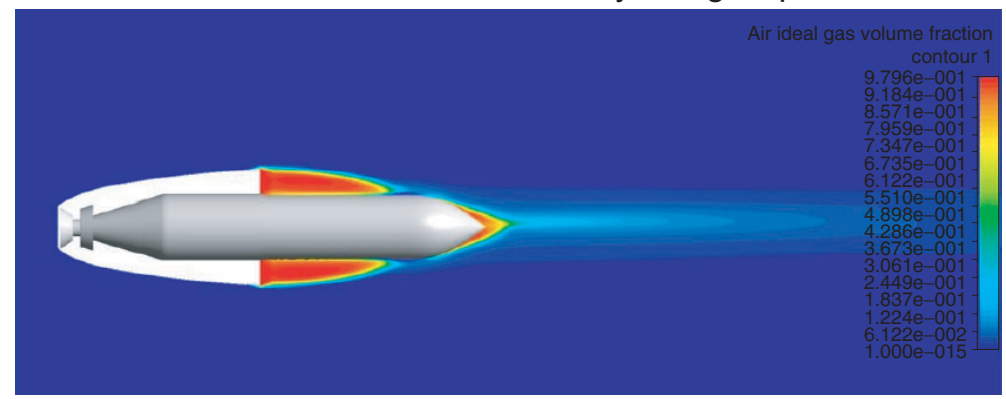

Figure 16. Prediction void fraction contour for Case 3.

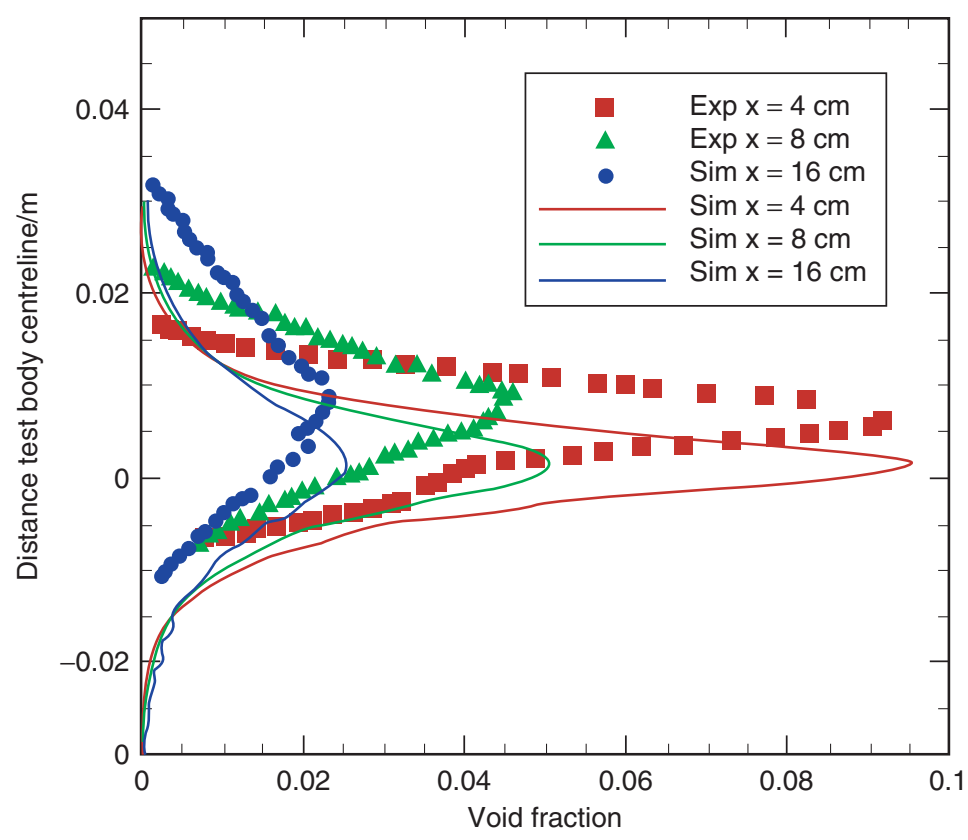

Figure 17. Void fraction in the bubbly wake (case 3).

were observed at the lower part of the cavity. This could be caused by the gravity effect which slightly shifted the cavity interface upward and reduced the shearing-off of bubbles from the lower cavity. The uniformly distribution gas injection could be in turn over-estimated the actually gas leakage rate from the lower side, causing over-prediction of void fraction in Figure 17.

\subsubsection{Influence of Froude Number}

Figure 18 shows the comparison of predicted void fraction distribution along the surface of the test body for the cavitating Case 2 and Case 3 . As depicted, the void fraction gradually decreases in the recirculation dominated area until the liquid re-attachment point. Another high void fraction area is also found at the tail of the test body. It can be observed that the predicted liquid re-attachment points were almost identical in both cases. This is agreed with the experiment observation that both cases show the same cavitation number 0.15 , determining the length of the cavity. The pressure coefficient distribution is presented in Figure 19. As the above, this parameter correlates well between two cases at the same cavitation number, especially in the recirculation dominated region. Comparison between the two cases also ascertained that a higher ventilation rate is required to achieve the same cavitation number for a low Froude number situation. 


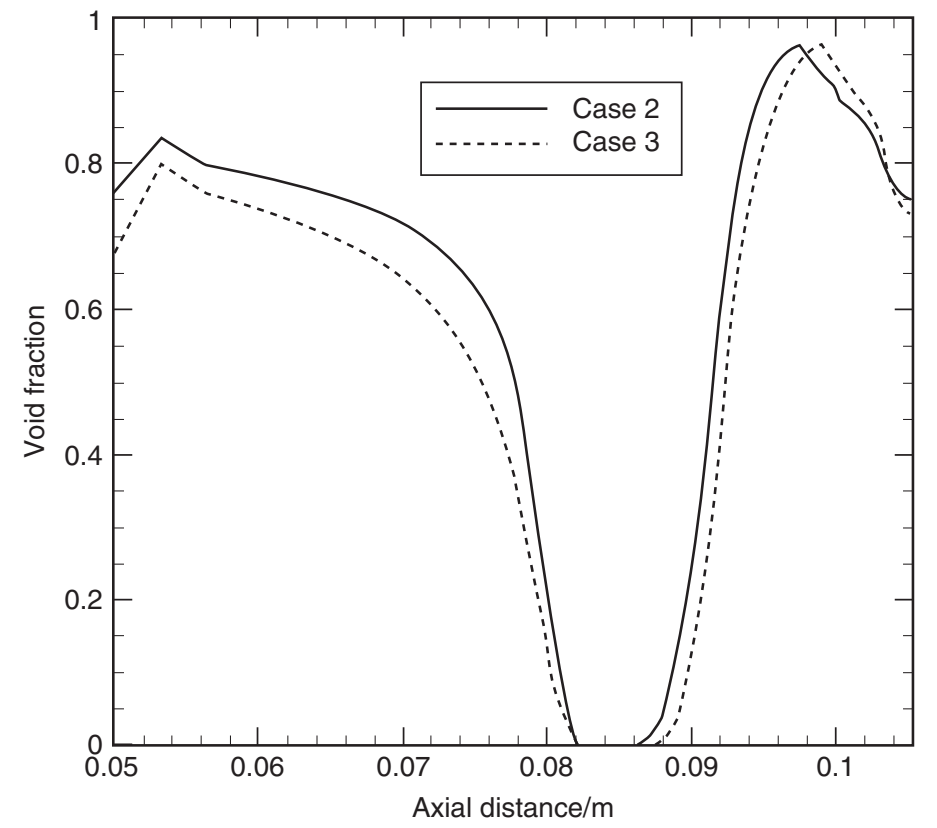

Figure 18. Void fraction distribution along the tail of the test body.

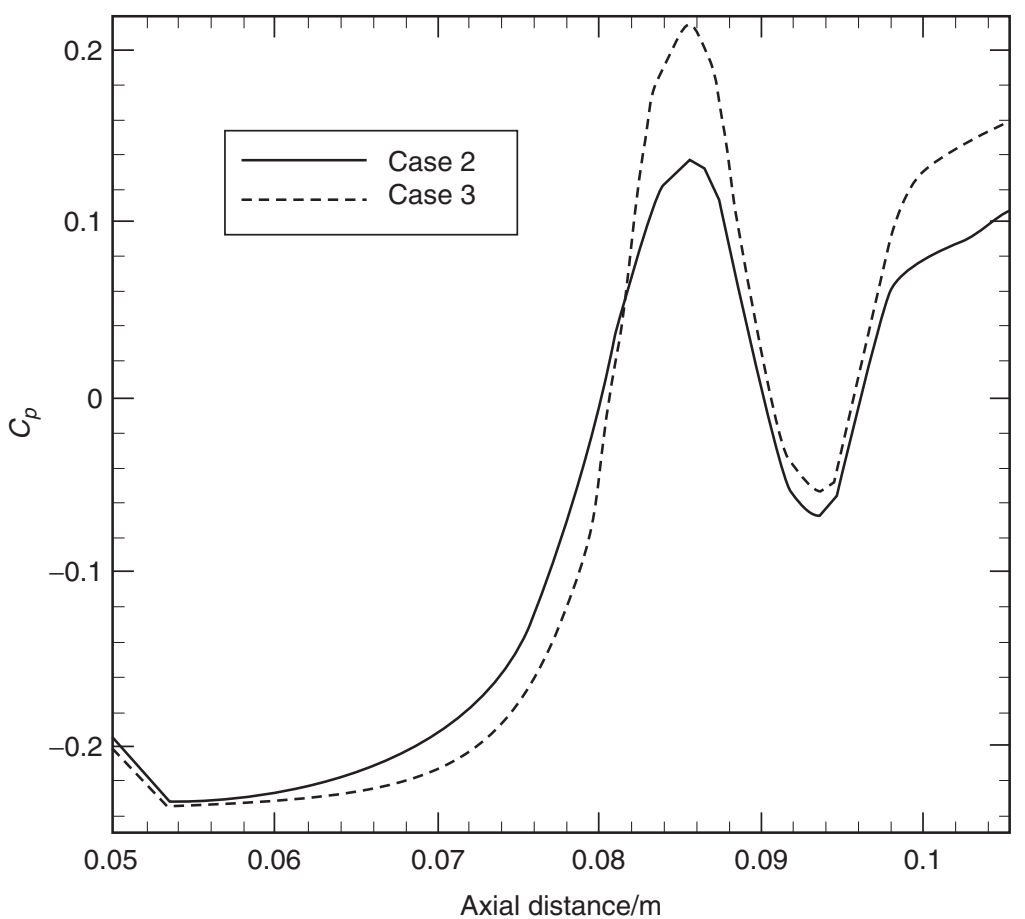

Figure 19. Pressure coefficient distribution along the tail of the test body.

\section{CONCLUSIONS}

The Eulerian-Eulerian two-fluid model, coupled with the population balance approach, is presented in this paper to handle the bubbly wake flow created of a Gilbarg-Efros type cavity closure. The interface of the ventilated cavity was determined by empirical equations of Logvinovich (1964) and Savchenko (1998). A sensitivity study on three turbulence modelling closures has demonstrated 
that the shear stress transport (SST) turbulence model is the best candidate in handling the recirculation motions within the wake region behind the cavity. Furthermore, to consider close packed bubble situation, an empirical equation is proposed to correlate the turbulent dispersion coefficient with local gas void fraction. Caused by the neighbouring effect of adjacent bubbles, the turbulent dispersion coefficient reduces to 0.1 if the local void fraction is higher than $60 \%$. In general, numerical predictions at various axial locations downstream of the test body were in satisfactory agreement with the experimental data. The treads of the radial void fraction profiles were successfully captured. Nonetheless, bubble velocities and the gravity effect on the void fraction distribution were slightly under-predicted. Numerical predictions also revealed that the liquid re-attachment lengths were almost identical in both cavitating cases and predominated by the Froude number. Such finding is agreed with the experimental observation that the cavitation numbers of both cases were found almost identical of 0.15 . This study presented a preliminary effort to incorporate population balance method into the two-fluid model to investigate the ventilated cavitating flow. Further effort should be also focused on combing free-surface model with the present population balance approach to investigate the complex vortex structure and interaction between continuous cavity and discrete dispersed leakage bubbles.

\section{REFERENCES}

Burns, A.D., Frank, T., Hamill, I., Shi, J.-M. (2004). The Favre averaged drag model for turbulenbce dispersion in Eulerian multi-phase flows. In: Proceedings of the Fifth International Conference on Multiphase Flow, ICMF, Yokohama, Japan.

Cheung, S.C.P., Yeoh G.H., Tu, J.Y., (2007). "On the numerical study of isothermal vertical bubbly flow using two population balance approaches”, Chem. Eng. Sci., 62, 4659-4674.

David, R.S. (2001). Basic research into high-speed supercavitating bodies. ONR project N00014-97-1-0126

Drew, D.A., Lahey Jr., R.T. (1979). Application of general constitutive principles to the derivation of multidimensional twophase flow equation. International Journal of Multiphase Flow, 5, 243-264.

Evans, G.M. (2004). Bubble Size Distribution and Void Fraction in the Wake Region Below a Ventilated Gas Cavity in Downward Pipe Flow. Chemical Engineering Research and Design. 82, 1095-1104.

Frank, T., Zwart, P.J., Shi, J. (2005). Inhomogeneous MUSIG model-a population balance approach for polydispersed bubbly flow. In: Proceeding of International Conference for Nuclear Energy for New Europe, Bled, Slovenia.

Goncalves, E. and Patella, R.F. (2009). Numerical Simulation of Cavitating Flows with Homogeneous Models. Computers \& Fluids, 38, 1682-1696.

Grace, J.R., Wairegi, T., Niguyen, T.H. (1976). Shapes and velocities of simple drops and bubbles moving freely through immiscible liquids. Trans. Inst. Chem. Eng, 54.

Hrubes, J.D. (2001). High-speed imaging of supercavitating underwater projectiles. Exp. Fluids, 30, 57-64.

Ishii, M., Zuber, N. (1979). Drag coefficient and relative velocity in bubbly, droplet or particulate flows. A.I.Ch.E. Journal, $5,843-855$.

Kinzel, M.P. and Lindau, J.W. (2008). Free-Surface Proximity Effects in Developed and Super-Cavitation, in DoD HPCMP Users Group Conference.

Kinzel, M.P. and Lindau, J.W. (2007). Computational Investigations of Air Entrainment, Hysteresis, and Loading for LargeScale, Buoyant Cavities, in HPCMP USERS GROUP CONFERENCE.

Kuklinski, R., Henoch, C., Castano J. (2001). Experimental study of ventilated cavities on dynamic test model, in: Proceedings of Fifth International Symposium on Cavitation (CAV2001), Pasadena, California, USA, Session B3.004.

Kunz, R.F. and Boger, D.A. (2000). A Preconditioned Navier-Stokes Method for Two-phase Flows with Application to Cavitation Predication. Computers and Fluids, 29, 849-875.

Kumar, S., Ramkrishna, D. (1996a). On the solution of population balance equations by discretisation-I. A fixed pivot technique. Chemical Engineering Science, 51, 1311-1332.

Launder, B., Salding, D. (1972). Lectures in Mathematical Models for turbulence. Academic Pres.

Lindau, J.W. and Kunz, R.F. (2005). Development and Application of Turbulent, Multiphase CFD to Supercavitation, in 2nd International Symposium on Seawater Drag Reduction. Busan, Korea.

Logvinovich G.V. (1969). Hydrodynamics of flows with free boundaries. - Kiev, Naukova dumka, 208, (in Russian) 
Luo, H., Svendsen, H. (1996). Theoretical model for drop and bubble break-up in turbulent dispersions. A.I.Ch.E. Journal, $42,1225-1233$.

Mandar, V.T., Swarnendu, A.R. (2008). CFD simulation of bubble column-An analysis of interphase forces and turbulence models. Chemical Engineering Journal, 139, 589-614.

Olmos, E., Gentric, C., Vial, C., Wild, G., Midoux, N. (2001). Numerical simulation of multiphase flow in bubble column. Influence of bubble coalescence and break-up. Chemical Engineering Science, 56, 6359-6365.

Owis, F.M. and Nayfeh A.H. (2002). A Compressible Multi-Phase Flow Solver for the Computation of the Super-Cavitation over High-Speed Torpedo, in AIAA-2002-0875.

Qin, Q. and Song C.C.S. (2003). A Numerical Study of an Unsteady Turbulent Wake Behind a Cavitating Hydrofoil, in Fifth International Symposium on Cavitation (Cav2003).

Sato, Y., Sadatomi, M., Sekoguchi, K. (1981). Momentum and heat transfer in two-phase bubbly flow-I. International Journal of Multiphase Flow, 7, 167-178.

Savchenko Yu.N., Vlasenko Yu.D., Semenenko V.N. (1998). Experimental study of high-speed cavitation flows. J. Hydromechanics., 72, 103-111, (in Russian)

Savchenko, Yu.N. (2001). Experimental Investigation of Supercavitating Motion of Bodies. VKI Special Course on Supercavitating Flows. Brussels_RTO-AVT and VKI, RTO-EN-010(4)

Schauer, T.J. (2003). An Experimental Study of a Ventilated Supercavitating Vehicle., MS Thesis in Aerospace Engineering, University of Minnesota.

Semenenko, V.N. (2001). Artificial Supercavitation. Physics and Calculation. VKI Special Course on Supercavitating Flows. Brussels_RTO-AVT and VKI_RTO-EN-010(11).

Simonnet, M., Gentric, C., Olmos, E., Midoux, N. (2007). Experimental determination of the drag coefficient in a swarm of bubbles, Chem. Eng. Sci, 62, 858-866

Tomiyama, A. (1998). Struggle with computational bubble dynamics. In: Proceeding of the Third International Conference on Multiphase Flow. Lyon, France.

Wosnik, M. and Fontecha, L.G. (2005). Measurements in High Void-fraction Bubbly Wakes Created by Ventilated Supercavitation, in Proceedings of FEDSM.

Yeoh, G.H., Tu, J.Y. (2004). Population balance modelling for bubbly flows with heat and mass transfer. Chemical Engineering Science, 59, 3125-3139.

Yeoh, G.H., Tu, J.Y. (2005). Thermal-hydrodynamic modelling of bubbly flows with heat and mass transfer. A.I.Ch.E. Journal, 51, 8-27.

Young, Y.L. and Kinnas S.A. (2003). Analysis of Supercavitating and Surface-piercing Propeller Flows via BEM. Computational Mechanics, 32, 4-6. 